\title{
Электролюминесценция в гетероструктурах $n$-GaSb/InAs/p-GaSb с одиночной квантовой ямой, выращенных методом МОГФЭ
}

\author{
() М.П. Михайлова, Э.В. Иванов, Л.В. Данилов, Р.В. Левин, И.А. Андреев, \\ Е.В. Куницына, Ю.П. Яковлев
}

Физико-технический институт им. А.Ф. Иоффре Российской академии наук, 194021 Санкт-Петербург, Россия

E-mail: Mayamikh@gmail.com

(Получена 17 июля 2018 г. Принята к печати 27 июля 2018 г.)

Сообщается об исследовании электролюминесцентных характеристик гетероструктуры II типа $n$-GaSb/n-InAs/p-GaSb с глубокой одиночной квантовой ямой, выращенной методом газофазной эпитаксии из металлоорганических соединений. Рассчитаны зонная энергетическая диаграмма структуры и положение уровней энергии электронов и тяжелых дырок. Из анализа вольт-амперных характеристик сделан вывод о туннельном механизме протекания темнового тока в изучаемой структуре. Обнаружена интенсивная электролюминесценция в спектральном диапазоне 3-4 мкм при $T=77$ и $300 \mathrm{~K}$, отличающаяся слабой температурной зависимостью. Основная полоса электролюминесценции $(h v=0.40$ эВ при $77 \mathrm{~K})$ соответствует прямым излучательным переходам между электронами с уровня $E_{1}$ в квантовой яме InAs и тяжелыми дырками континуума на гетерогранице $n$-GaSb $/ n$-InAs. Малоинтенсивный пик $(h v=0.27$ эB, $T=77 \mathrm{~K})$ обусловлен непрямыми (туннельными) переходами с первого уровня электронов в квантовой яме на второй уровень тяжелых дырок, локализованных в разрыве валентной зоны на гетерогранице $n$-InAs/ $p$-GaSb.

DOI: 10.21883/FTP.2019.01.46986.8958

\section{1. Введение}

Гетероструктуры на основе InAs/GaSb представляют большой интерес для исследований благодаря уникальной разъединенной гетерогранице II типа, поскольку дно зоны проводимости InAs располагается на 150 мэВ ниже потолка валентной зоны GaSb [1]. Зонная диаграмма квантовых ям (КЯ) в системе InAs/GaSb трансформируется из полуметаллической в полупроводниковую в том случае, когда ширина КЯ $d<8.5$ нм [2]. Такие гетероструктуры перспективны для создания инфракрасных светодиодов [3], фотодиодов [4, 5] и квантово-каскадных лазеров на основе сверхрешеток [6].

Важным преимуществом светоизлучающих структур с глубокими КЯ является то, что в них ожидается существенное подавление безызлучательной ожерекомбинации в диапазоне значений ширины КЯ 4.5-10 нм [7]. В работе было показано, что наличие гетерограницы снимает ограничения на закон сохранения продольной компоненты квазиимпульса, что приводит к появлению беспороговых, слабо зависящих от температуры каналов оже-рекомбинации. Особенно значим этот процесс в гетеропереходах II типа с КЯ.

Ранее в работах [8-10] были исследованы электролюминесцентные свойства гетероструктур II типа на основе полупроводниковых соединений InAs и $\mathrm{GaSb}$. В [8] изучались гетероструктуры $n$-InAs/ $N-\mathrm{GaSb}$ и $n$-In $(\mathrm{Ga}) \mathrm{As}(\mathrm{Sb}) / N-\mathrm{GaSb}$ с самосогласованными КЯ, выращенные методом газофазной эпитаксии из металлоорганических соединений (МОГФЭ) и методом жидкофазной эпитаксии (ЖФЭ) соответственно. Установлено, что изотипные структуры $\mathrm{GaSb} / \mathrm{In}(\mathrm{Ga}) \mathrm{As}(\mathrm{Sb})$ с узкозонным слоем твердого раствора, обогащенным арсени- дом индия, являются разъединенными гетеропереходами II типа. Электролюминесценция (ЭЛ) наблюдалась в спектральном диапазоне 3-4 мкм при температуре $77 \mathrm{~K}$. Излучательные переходы при прямом смещении имели место вблизи разъединенной $N-n$-гетерограницы. Было показано, что сильное перекрытие волновых функций электронов и дырок, расположенных в самосогласованных КЯ на $N-n$-интерфейсе, приводит к непрямой излучательной рекомбинации через гетерограницу, сопровождающейся туннелированием носителей.

В работе [9] была исследована излучательная рекомбинация в разъединенной гетероструктуре II типа $p$-GaInAsSb/p-InAs, выращенной методом ЖФЭ, в температурном диапазоне $4-100 \mathrm{~K}$. Обнаружено, что в такой структуре при $T=77 \mathrm{~K}$ на интерфейсе образуется электронный канал с высокой подвижностью носителей $\left(\mu \geq 5 \cdot 10^{4} \mathrm{~cm}^{2} / \mathrm{B} \cdot \mathrm{c}\right)$. В спектрах ЭЛ присутствовали две полосы, одна из которых $\left(h v_{A} \approx 0.37\right.$ эВ) была обусловлена рекомбинацией электронов из полуметаллического канала с участием глубокого акцепторного уровня, а вторая полоса $\left(h v_{B} \approx 0.40\right.$ эВ) соответствовала излучательной рекомбинации в объеме InAs. Было показано, что ЭЛ возникала благодаря непрямой (туннельной) рекомбинации электронов и дырок, локализованных в самосогласованных КЯ, через гетерограницу GaInAsSb/InAs.

Влияние гетерограницы на непрямые оптические переходы в полупроводниковых квантовых структурах теоретически рассматривалось в работе [11]. Было показано, что в гетероструктурах с КЯ оптические переходы всегда непрямые (в пространстве импульсов). Это приводит к тому, что оптическое усиление для непрямых переходов всегда выше, чем для прямых из-за более 
высокой плотности состояний. При этом температурная зависимость для непрямых переходов слабее, чем для прямых.

Электролюминесцентные свойства наногетероструктур с короткопериодными сверхрешетками на основе разъединенных гетеропереходов II типа InAs/GaSb, выращенных методом молекулярно-пучковой эпитаксии на подложках $p$-InAs(100), изучались в работе [10]. ЭЛ наблюдалась в диапазоне 3-4мкм при температуре $77 \mathrm{~K}$. Для трехпериодных гетероструктур 50 нмInAs/10 нм-GaSb в спектрах ЭЛ, как и в предыдущей работе [9], присутствовали две полосы с максимумами в районе 0.36 и 0.41 эВ. Существование длинноволновой полосы ЭЛ было связано с непрямыми (туннельными) излучательными переходами на гетерогранице II типа $\mathrm{GaSb} / \mathrm{InAs}$, когда электроны, локализованные на уровне размерного квантования в КЯ со стороны InAs, рекомбинируют с тяжелыми дырками в $\mathrm{GaSb}$. При исследовании трехпериодной гетероструктуры 5 нм-InAs/5 нм$\mathrm{GaSb}$, помещенной между сильно легированными $p^{+}$- и $n^{+}$-слоями 5 нм-InAs и широкозонными слоями 10 нм$\mathrm{AlSb}$, в спектрах ЭЛ были обнаружены три полосы, связанные с излучательными переходами электронов с трехкратно расщепленного уровня в решетке InAs/GaSb на уровни локализации тяжелых дырок.

Таким образом, в исследованных ранее гетероструктурах наблюдалась ЭЛ, обусловленная рекомбинационными переходами с участием электронов с уровней размерного квантования как из самосогласованных КЯ на гетерогранице, так и из прямоугольных КЯ в короткопериодных решетках.

В данной работе мы сообщаем о первом исследовании электролюминесцентных свойств гетероструктур II типа $n$-GaSb/n-InAs/p-GaSb с одиночной КЯ, выращенных методом МОГФЭ.

\section{2. Технология изготовления структур}

Исследуемые структуры были выращены на установке AIXTRON 200 с горизонтальным реактором на подложке $n-\mathrm{GaSb}(001)$ при температурах $600^{\circ} \mathrm{C}$ для слоев $\mathrm{GaSb}$ и $500^{\circ} \mathrm{C}$ для КЯ InAs. Давление в реакторе составляло 76 Topp. В процессе роста подложка вращалась со скоростью 100 об/мин. В качестве газа-носителя использовался очищенный водород. Источниками элементов для роста (прекурсорами) являлись триметилиндий (TMIn), триэтилгаллий (TEGa), триметилсурьма (TMSb) и арсин $\left(\mathrm{AsH}_{3}\right)$. Диэтилтеллур $\left(\mathrm{DET}_{e}\right)$ и силан $\left(\mathrm{SiH}_{4}\right)$ использовались в качестве источников легирующих примесей доноров и акцепторов соответственно. Соотношение элементов V и III групп имело следующие значения: $\mathrm{AsH}_{3} / \mathrm{TMIn}=93$ и TMSb/TEGa $=2$. Методика выращивания подробно описана в работе [12].

Изучаемая структура состояла из подложки $n$-GaSb:Te $\quad\left(n=(5-7) \cdot 10^{17} \mathrm{~cm}^{-3}\right), \quad$ буферного слоя $n$-GaSb:Te (толщина $0.8-0.9$ мкм, $n=5 \cdot 10^{17} \mathrm{~cm}^{-3}$ ), нелегированной одиночной КЯ InAs $(d=5$ нм $)$ и накрывающего слоя $p$ - $\mathrm{GaSb}: \mathrm{Si}$ (толщина 1 мкм, $\left.p=(3-4) \cdot 10^{17} \mathrm{~cm}^{-3}\right)$. Образцы для измерений были изготовлены методом стандартной фотолитографии и мокрого травления в виде мезадиодов с диаметром $\sim 300$ мкм. Омические контакты были сформированы путем вакуумного напыления сплавов $\mathrm{Cr} / \mathrm{Au}+\mathrm{Te} / \mathrm{Au}$ и $\mathrm{Cr} / \mathrm{Au}+\mathrm{Ge} / \mathrm{Au}$ к подложке $n$-GaSb и накрывающему слою $p$-GaSb соответственно.

\section{3. Методика эксперимента}

Спектры ЭЛ гетероструктур $n$-GaSb/InAs/p-GaSb были исследованы в диапазоне 2.5-5.5 мкм с помощью установки на основе монохроматора DK-480 с дифракционной решеткой 150 штрих/мм (CVI Laser Corp.) при температуре 77 и 300 К. Исследовалось излучение, выходящее из структуры со стороны накрывающего слоя $p$-GaSb. ЭЛ возбуждалась прямоугольными импульсами тока с коэффициентом заполнения 50\% (меандр) и частотой 512 Гц. Амплитуда импульсов варьировалась в пределах от 20 до 200 мА. В качестве приемника излучения использовался фотовольтаический детектор InSb, paботающий при температуре жидкого азота (Judson Co). Фотосигнал детектировался при помощи селективного усилителя SR-810 (Stanford Research Systems Inc.) и передавался в компьютер. Для того чтобы избежать наложения спектров высшего порядка от коротковолновой составляющей ЭЛ из подложки $n-\mathrm{GaSb}$, использовался интерференционный фильтр, отрезающий излучение с длиной волны менее 2.5 мкм.

Вольт-амперные характеристики исследовались с помощью автоматизированной компьютерной установки в диапазоне температур 77-300 K.

\section{4. Результаты и обсуждение}

Вольт-амперные характеристики (BAX) изучаемых гетероструктур при температурах 77 и $300 \mathrm{~K}$ показаны на рис. 1. Величина напряжения отсечки в прямой ветви составляла $\sim 0.4$ и $\sim 0.5$ В для комнатной температуры и для температуры жидкого азота соответственно. Проведенный анализ показал, что прямые ветви ВАХ состоят из трех участков, каждый из которых может быть аппроксимирован экспоненциальной зависимостью $I=I_{0} \exp (e U / \beta k T)$. При этом величина коэффициента неидеальности была равна $\beta=3-7(T=300 \mathrm{~K})$ и $\beta=14-42(T=77 \mathrm{~K})$, что соответствовало туннельному механизму протекания тока. Нелинейный характер BAX может быть объяснен тем, что полный ток через структуру представляет собой сумму токов электронов и дырок, включающую вклад как туннельной, так и термоэмиссионной составляющих.

На рис. 2 представлены спектральные характеристики ЭЛ при температурах 77 и $300 \mathrm{~K}$ в зависимости от тока накачки в диапазоне 20-200 мА для случая прямой 


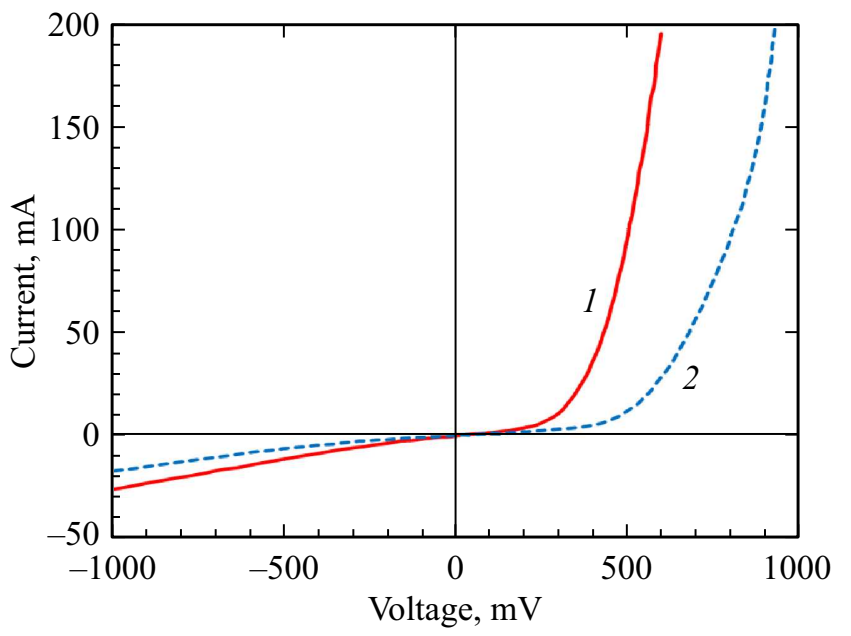

Рис. 1. Вольт-амперная характеристика гетероструктуры $n$-GaSb/InAs/p-GaSb при температурах 300 (1) и $77 \mathrm{~K}(2)$.
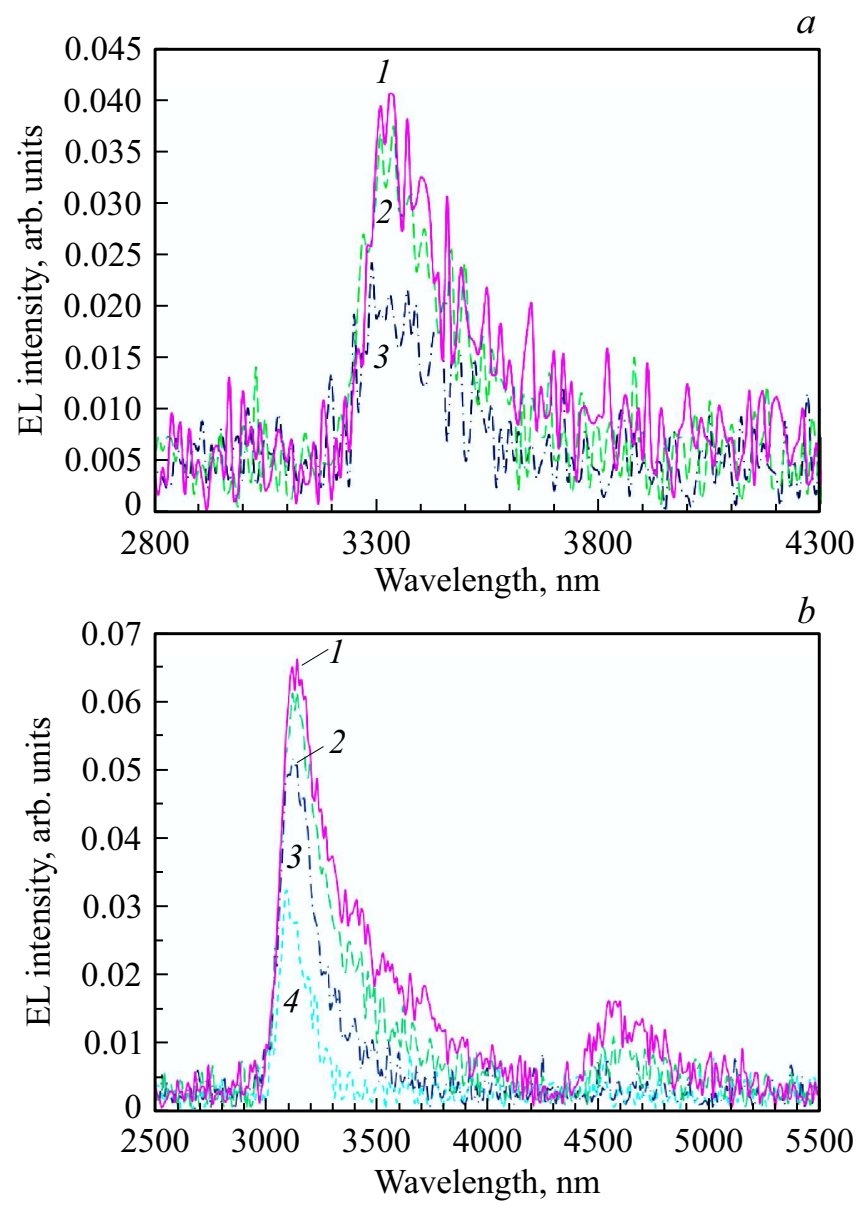

Рис. 2. Спектры электролюминесценции структуры $n$-GaSb/InAs/ $p$-GaSb при $T=300(a)$ и $77 \mathrm{~K}(b)$ для различных значений тока накачки, мА: $1-150,2-100,3-50$, $4-25$.

полярности внешнего смещения, когда отрицательный потенциал был приложен к подложке $n$-GaSb, а положительный - к накрывающему слою $p$-GaSb. ЭЛ воз- никала при приложении прямого смещения $\sim 0.35 \mathrm{~B}$ $(T=300 \mathrm{~K})$ и $\sim 0.45 \mathrm{~B}(T=77 \mathrm{~K})$, что приблизительно равно значениям напряжения отсечки в прямой ветви BAX при соответствующих температурах.

В спектрах ЭЛ наблюдалась интенсивная асимметричная полоса в диапазоне длин волн 3-4 мкм с резким коротковолновым и пологим длинноволновым краями. При $T=300 \mathrm{~K}$ (см. рис. 2, $a$ ) максимум спектров ЭЛ соответствовал длине волны $3.32 \mathrm{Mкм}(h v=0.37$ эВ), а полуширина спектров составляла 20-25 мэВ. При температуре жидкого азота (рис. 2,b) длина волны в максимуме излучения для основной полосы ЭЛ была $\sim 3.1$ мкм $(h v=0.40$ эВ). Для токов накачки, не превышающих 50 мА, полуширина данной полосы была такой же, как при $T=300 \mathrm{~K}$. Наблюдавшаяся в эксперименте температурная зависимость максимума спектра ЭЛ соответствовала коэффициенту $\Delta(h v) / \Delta T=-0.135$ мэВ $/ \mathrm{K}$. По абсолютной величине данное значение вдвое меньше параметра $\Delta E_{g} / \Delta T=-0.27$ мэВ $/ \mathrm{K}$, характерного для арсенида индия [13].

При комнатной температуре форма спектров ЭЛ, их полуширина и положение максимума практически не изменялись во всем диапазоне токов накачки от 20 до 200 мА. При $T=77 \mathrm{~K}$ максимум спектров ЭЛ слабо смещался в длинноволновую сторону при увеличении накачки, по-видимому, вследствие нагрева исследуемой структуры. При высоких значениях тока ( $\geq 100 \mathrm{MA})$ в спектре ЭЛ появлялось дополнительное „плечо“ в окрестности длины волны 3.55 мкм (0.35 эВ). Кроме того, при температуре жидкого азота в спектрах ЭЛ наблюдалась вторая, слабая по интенсивности полоса с максимумом около 4.6 мкм (0.27 эВ).

На рис. 3 показана зависимость интенсивности основной полосы в спектре ЭЛ от тока накачки для температур 77 и $300 \mathrm{~K}$. Обе зависимости являются сублинейными и при токе свыше 100 мА выходят на уровень насыщения. При этом максимальные значения

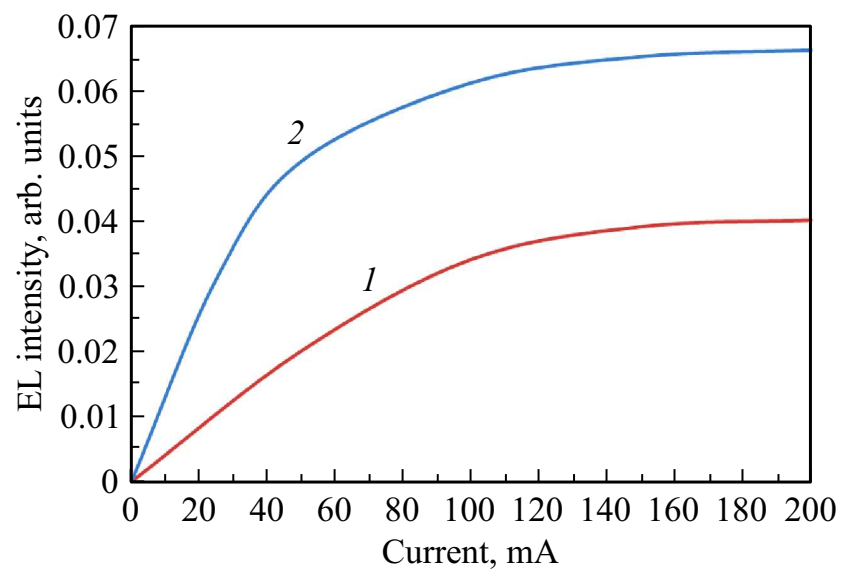

Рис. 3. Зависимость интенсивности основной полосы электролюминесценции от тока накачки для структуры $n$-GaSb/InAs/p-GaSb при температурах 300 (1) и $77 \mathrm{~K}(2)$. 


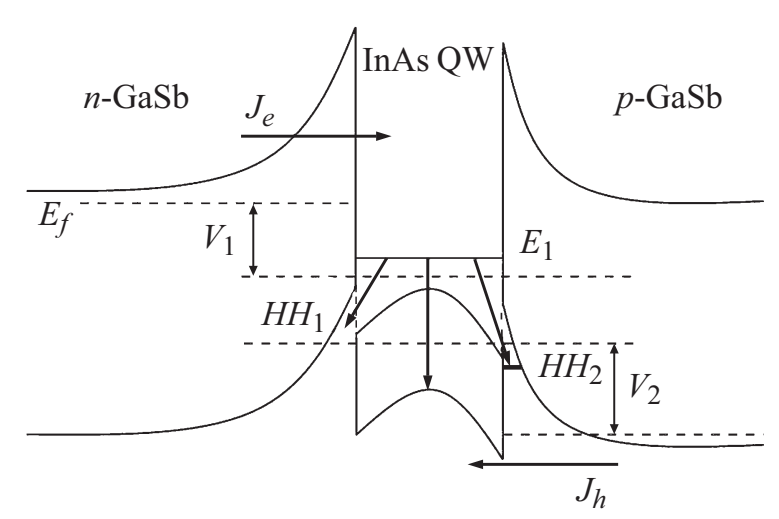

Рис. 4. Зонная энергетическая диаграмма гетероструктуры $n$-GaSb/InAs/p-GaSb при прямом смещении $V=V_{1}+V_{2} . E_{\mathrm{F}}-$ уровень Ферми, $E_{1}-$ основной уровень энергии электронов в КЯ InAs, $H_{1}$ и $H_{2}$ - первый и второй уровни тяжелых дырок соответственно.

интенсивности, достигнутые при $T=77$ и $300 \mathrm{~K}$, различались всего в $1.5-2$ раза.

В случае обратного смещения (когда к подложке $n$-GaSb был приложен положительный потенциал) ЭЛ в исследованных структурах не наблюдалась.

Для анализа экспериментальных результатов нами был проведен теоретический расчет зонной диаграммы и энергетических уровней электронных и дырочных состояний в рассматриваемой структуре с использованием численного самосогласованного решения уравнений Шредингера и Пуассона. Как видно из рис. 4, зонная диаграмма исследуемых структур представляла собой два разъединенных гетероперехода II типа, включенных навстречу друг другу: $n$-GaSb/n-InAs и $n$-InAs/ $p$-GaSb. Расчет уровней размерного квантования дырок был проведен в рамках квазиклассического приближения для треугольной КЯ [3]. В этом случае энергия дырок зависит от напряженности приложенного электростатического поля $F$ по закону $\sim F^{2 / 3}$.

Согласно расчету, при нулевом внешнем смещении энергия основных уровней электронов и тяжелых дырок в КЯ InAs равна $E_{1}=0.2$ эВ и $H H_{1}=0.07$ эВ соответственно (см. рис. 4). При этом уровни дырок $H_{1}$ и $H_{2}$ располагаются в разрывах валентной зоны на интерфейсах $n$-GaSb/n-InAs и $n$-InAs/ $p$-GaSb, соответственно. Таким образом, энергия непрямого (туннельного) оптического перехода $E_{1}-H H_{1}$ между первым электронным и первым дырочным уровнями составляет $\left(E_{1}+H H_{1} \sim \Delta\right)$, где $\Delta=150 \mathrm{мэВ,} \mathrm{равна} \mathrm{расстоянию} \mathrm{от}$ дна зоны проводимости InAs до потолка валентной зоны GaSb. Энергия кванта, соответствующего данному переходу, равна 0.12 эВ (т.е. $\lambda=10.3$ мкм). Положение основного уровня размерного квантования электронов $E_{1}$ и второго уровня тяжелых дырок $H_{2}$, а также энергия излучательного перехода $E_{1}-H H_{2}$ сильно зависят от изгиба зон на гетерогранице $n$-InAs/ $p-\mathrm{GaSb}$, возникающего при приложении прямого смещения. В этом случае энергия перехода $E_{1}-H_{2}$ может быть записана как $\left(E_{1}+H H_{2}-\Delta+e \varphi\right)$, где $\varphi-$ электростатический потенциал, приложенный к слою InAs. Вследствие этого энергия непрямого оптического перехода $E_{1}-H_{2}$ оказывается больше, чем энергия перехода $E_{1}-H H_{1}$, что согласуется с экспериментальными спектрами ЭЛ.

Полоса ЭЛ в окрестности 0.37 ЭВ при $T=300 \mathrm{~K}$ и 0.40 эВ при $T=77 \mathrm{~K}$ соответствует излучательным рекомбинационным переходам между электронами из основного энергетического состояния $E_{1}$ в КЯ InAs и тяжелыми дырками континуума на гетерогранице $n$-GaSb/n-InAs. Возникновение длинноволнового „плеча“ в спектрах ЭЛ при температуре жидкого азота по мере роста тока накачки можно объяснить оптическими переходами из варизонных слоев твердого раствора $\operatorname{InAs}_{1-x} \mathrm{Sb}_{x}$, которые сформировались в процессе выращивания структуры методом МОГФЭ вблизи интерфейсов КЯ InAs [14]. Мы полагаем, что дополнительный малоинтенсивный пик в районе 0.27 эВ при $T=77 \mathrm{~K}$ соответствует рекомбинационному переходу $\mathrm{E}_{1}-\mathrm{HH}_{2}$, в котором участвуют тяжелые дырки, локализованные вблизи гетерограницы в валентной зоне $p$-GaSb при прямом смещении. При этом длинноволновая полоса, связанная с переходом $E_{1}-H H_{1}(\lambda=10.3$ мкм), располагается за пределами спектрального диапазона чувствительности фотодетектора InSb, использованного в процессе измерений.

Тот факт, что ЭЛ не наблюдалась, когда к исследуемой структуре было приложено обратное смещение, объясняется отсутствием тока электронов через гетерограницу $n$-InAs $/ n-\mathrm{GaSb}$, что связано с особенностями зонной диаграммы данного изотипного гетероперехода [9].

\section{5. Заключение}

Впервые выращена гетероструктура $n$-GaSb/n-InAs/ $p$-GaSb с одиночной КЯ InAs методом МОГФЭ. Рассчитаны зонная энергетическая диаграмма структуры, представляющая собой два разъединенных гетероперехода на интерфейсах КЯ, и положение уровней энергии для электронов и тяжелых дырок. При приложении прямого смещения наблюдалась интенсивная ЭЛ в диапазоне длин волн $3-4$ мкм при $T=77$ и $300 \mathrm{~K}$. Показано, что основная полоса ЭЛ $(h v=0.37$ эВ при $T=300 \mathrm{~K}$ и 0.40 эВ при $77 \mathrm{~K}$ ) обусловлена прямыми излучательными переходами между электронами с уровня $E_{1}$ в КЯ InAs и тяжелыми дырками континуума на гетерогранице n$\mathrm{GaSb} / n$-InAs. При $T=77 \mathrm{~K}$ в спектре ЭЛ наблюдался дополнительный малоинтенсивный пик $(h v=0.27$ э), соответствующий туннельному (непрямому) переходу с основного уровня электронов в КЯ на второй уровень тяжелых дырок, локализованных в разрыве валентной зоны на гетерогранице $n$-InAs/ $p$-GaSb. Слабая температурная зависимость спектра ЭЛ согласуется с теоретическими представлениями о роли беспороговых процессов оже-рекомбинации в глубоких КЯ. ВАХ гетерострукту- 
ры подтверждает вывод о туннельном механизме протекания темнового тока.

Исследованная наноструктура представляет интерес с точки зрения создания светоизлучающих диодов, работающих в среднем инфракрасном диапазоне в широком интервале температур от 77 до $300 \mathrm{~K}$.

Работа частично поддержана проектом президиума РАН и грантом РФФИ № 17-52-16029-НЦНИЛа.

\section{Список литературы}

[1] G.A. Sai-Halasz, L. Esaki, W.A. Harrison. Phys. Rev. B, 18 (6), 2812 (1978).

[2] M. Altarelli, J.C. Maan, L.L. Chang, L. Esaki. Phys. Rev. B, 35 (18), 9867 (1987).

[3] M. Mikhailova, N. Stoyanov, I. Andreev, B. Zhurtanov, S. Kizhaev, E. Kunitsyna, K. Salikhov, Yu. Yakovlev. Proc. SPIE, Optical Sensing Technology and Applications, 6585 658526 (2007).

[4] M. Razeghi, D. Hoffman, B.-M. Nguyen, P.-Y. Delaunay, E.K. Huang, M.Z. Tidrow. Proc. SPIE, Infrared Technology and Applications XXXIV, 6940, 694009 (2008).

[5] М.П. Михайлова, И.А. Андреев, К.Д. Моисеев, Э.В. Иванов, Г.Г. Коновалов, М.Ю. Михайлов, Ю.П. Яковлев. ФТП, 45 (2), 251 (2011).

[6] R. Teissier, D. Barate, Y. Devinson, A.N. Baranov, X. Marcadet, C. Bernard, C. Sirtori. Inst. Phys. Conf. Ser., 187, 307 (2005).

[7] Л.В. Данилов, Г.Г. Зегря. ФТП, 42 (5), 566 (2008).

[8] K.D. Moiseev, M.P. Mikhailova, N.D. Stoyanov, Yu.P. Yakovlev, E. Hulicius, T. Simecek, J. Oswald, J. Pangrac. J. Appl. Phys., 86 (11), 6264 (1999).

[9] К.Д. Моисеев, М.П. Михайлова, Ю.П. Яковлев, И. Освальд, Э. Гулициус, И. Панграц, Т. Шимечек. ФТП, 37 (10), 1214 (2003).

[10] К.Д. Моисеев, Б.Я. Мельцер, В.А. Соловьев, С.В. Иванов, М.П. Михайлова, Ю.П. Яковлев, П.С. Копьев. Письма ЖТФ, 24 (12), 50 (1998).

[11] G.G. Zegrya, M.Yu. Mikhailov. Abstracts Int. Symp. „Nanostructures: Physics and Technology" (St. Petersburg, Russia, June 1994) p. 100.

[12] Р.В. Левин, В.Н. Неведомский, Б.В. Пушный, Н.А. Берт, М.Н. Мизеров. Письма ЖТФ, 42 (2), 79 (2016).

[13] Handbook Series of Semiconductor Parameters, ed. by M. Levinstein, S. Rumyantsev, M. Shur (SingaporeN. Y.-London-Hong Kong, World Scientific Publishing, 1996) v. 1.

[14] N. Bertru, A.N. Baranov, Y. Cuminal, G. Boissier, C. Alibert, A. Joullie, B. Lambert. J. Appl. Phys., 85 (3), 1989 (1999).

\section{Electroluminescence in $n$-GaSb/InAs/p-GaSb heterostructures with a single quantum well grown by MOVPE}

M.P. Mikhailova, E.V. Ivanov, L.V. Danilov, R.V. Levin, I.A. Andreev, E.V. Kunitsyna, Yu.P. Yakovlev

loffe Institute, 194021 St. Petersburg, Russia

\begin{abstract}
The paper reports on study of electroluminescent properties of the type II $n$-GaSb/n-InAs/ $p$-GaSb heterostructure with a single deep quantum well grown by metal organic vapor phase epitaxy. Band energy diagram of the structure and energy states of electrons and heavy holes were calculated. From analysis of the current-voltage characteristics the conclusion about tunneling mechanism of dark current flow in the structure was deduced. Intensive electroluminescence characterized by weak temperature dependence was found in the spectral range of $3-4 \mu \mathrm{m}$ at $T=77$ and $300 \mathrm{~K}$. Main electroluminescence band $(h v=0.40 \mathrm{eV}$ at $77 \mathrm{~K}$ ) corresponds to direct radiation transitions between electrons from $E_{1}$ level in InAs quantum well and heavy holes from continuum at $n$-GaSb/n-InAs heterointerface. Low intensive electroluminescence band $(h v=0.27 \mathrm{eV}, T=77 \mathrm{~K})$ is due to indirect (tunneling) transitions from the first electron level in the quantum well to the second level of heavy holes localized in the valence band offset at the $n$-InAs/ $p$-GaSb heterointerface.
\end{abstract}

